\title{
The IAU Role
}

\author{
Oddbjørn Engvold \\ Institute of Theoretical Astrophysics, University of Oslo, Oslo, Norway \\ email: oengvold@astro.uio.no
}

\section{Introductory Comments}

One knows currently close to 850 Near Earth Asteroids (NEAs) with diameters $1 \mathrm{~km}$ and larger, and one estimates that there may be of the order of 100000 NEAs with diameters exceeding $140 \mathrm{~m}$. Land and water impacts of NEAs with diameters between $100 \mathrm{~m}$ and $500 \mathrm{~m}$ will cause major damages.

Governments and international organizations are becoming increasingly concerned with natural hazards and disasters. The International Council for Science (ICSU) is developing a new program on "Natural and human-induced hazards and disasters" with participation from a large number of its scientific Unions. An adequate survey of Near Earth Objects (NEOs) is of notable interest and importance in such an initiative

A rapid development of NEOs survey programs illustrates the increased interest for detecting, tracking, characterizing and cataloging this family of bodies in our solar system. The first of four 2-m telescopes for such a survey program, the University of Hawaii Panoramic Survey Telescope and Rapid Response System (Pan-STARRS), will be operative in 2007. The planned Large Synoptic Survey Telescope (LSST) in Cerro Pachon, Chile, will further enhance the discovery rate of NEOs. One may also note that NASA has recently modified its charter to stress its increased responsibility for discovery and characterization of NEOs.

The discoveries of NEAs that will be "interesting" to the public and media may increase from about one per year to very possibly one per week. Based on preliminary orbit calculations, as much as a dozen of these may initially appear to pose a potential threat.

The IAU has been and will continue to be the primary international scientific organization with expertise on NEOs.

\section{IAU's Responsibilities and Initiatives}

The greatly increased discovery rate of NEAs will inevitably lead to an increased interest in characterization and studies of them. Calculation of precise orbits and determination of impact probabilities for potentially hazardous NEAs will in the very near future require a matching attention and augmentation in support. The IAU has an obligation to encourage its National Members to support and safeguard these important scientific activities.

The IAU has for several decades assumed responsibility for the IAU Minor Planet Center hosted at Smithsonian Astrophysical Observatory (SAO) with the important task of recording and maintaining the inventory of small bodies in the solar system, which constitutes an important service to our community at large. A new Memorandum of Agreement between the IAU and SAO was signed in June 2006, which will ensure continued operation of the MPC with funding from NASA. The IAU is pleased to note SAO's willingness and intention to revise and upgrade the operations of the MPC in order to meet the need to cope with up to a hundred-fold increase in incoming data. 
One byproduct of the increasingly improved NEO surveys will be the finding of many new faint comets which concerns the IAU through their scientific value and interest.

With its responsibility for safeguarding and coordination of the science of astronomy in all its aspects IAU is the obvious organization and authority to be undertaking a sober and quality controlled information, on the matter of potential NEA threats to the Earth, to the public, to media and to governments. The IAU Executive Committee has created a small committee on "Impact Threats on the Earth" to enable the IAU President and General Secretary to respond quickly to media and the public, as well as to governmental and international organizations, on incidents of Near-Earth asteroids and comets.

The current members of this Advisory Committee are: David Morrison (Chair), Richard Binzel, Andrea Carusi, Andrea Milani, Donald Yeomans and the Director of IAU Minor Planet Center. The Committee will conduct its duties in accordance with the following rules and restrictions:

- Committee members will keep each other informed and will share information.

- Non-urgent communications to the IAU will be based on committee consensus.

- Urgent communications should represent a committee consensus, but can be initiated by a minimum of 2 (two) committee members .

- The committee will establish a public web page to be used for both general and urgent information. The site will cross-link to the official IAU web site and to the primary CEO web site (JPL, Pisa, MPC, etc.).

- Urgent statements on behalf of the IAU can be posted by a minimum of 2 committee members. All urgent messages will also be sent by e-mail to the IAU GS, President and Press Officer.

- The NEO web page will provide a historical record of IAU statements, including, for example, both original statements and subsequent modifications.

- The Committee will not issue IAU press releases or hold press conferences, but it will assist IAU in such press statements, if requested.

- Interaction with governments on issues of impact threats is under the sole control of the IAU President and General Secretary.

\section{Acknowledgements}

I thank David Morrison for helpful input to my preparation of this brief intervention.

\section{Discussion}

HARRIS: We had a small committee (the Technical Review Committee) that backfired on us because it was taking the data about possible collisions and was evaluating them essentially in secret, in order to advise the IAU General Secretary and the public at large. I would rather see things to operate as Steve Chesley said yesterday in his talk, with the impact monitoring groups posting their results on the web, when the results agree, leaving to the public to judge. I still see a very valuable use for the new committee, that is to provide expert evaluation about the problem to the IAU and to the general public.

VALSECCHI: You were speaking of the Technical Review Committee; that committee was established because at the beginning of impact monitoring there was only one group doing the job. The function of the committee was superseded by the existence of a second, independent impact monitoring system, witn the ensuing cross-checking. Concerning secrecy, let me say that when you have a confirmed news, and you do not disseminate it, then that is secrecy. However, when you are not yet convinced of the correctness of what you have found, and are trying to understand whether it is reliable, then that is 
not secrecy, that is checking. We (i.e., the community involved) were convinced from the beginning that secrecy backfires; at the same time, we wanted to be sure that what we had found was news worthy of being communicated. In the case of (99942) Apophis the information was released after three days of checking, since the initial data were of such bad quality that we could not trust the results that were coming out of the impact monitoring systems; as soon as we cleared all our doubts, the information was immediately released.

Milani: I would like to comment on the recurring issues of verification and secrecy. First of all, we do wait for confirmation of all the most important (Palermo Scale > $-2)$ possible impacts. To understand how, you have to take into account time zones: there is a difference of 9 hours in local time between the location of the two current Impact Monitoring Systems, CLOMON2 in Europe and Sentry in California. Verification typically takes a short time, but in critical cases requires that the two teams are in their offices in the same moment, which is possible only in some afternoon hours (European time). As soon we are convinced the data have been verified by cross checking between the two systems we post immediately, within minutes. This is the situation now, and it is a very significant improvement with respect the time (before 2002) when only one impact monitoring system was available. Indeed, with duplication we have solved the problem of verification. This gives an important guarantee of reliability and also has relieved a lot of stress, which used to affect the previously unique team.

Another important point is that we do operate only on observational data which are public. Moreover, the algorithms and software we use for this are public and available on the web. The OrbFit software system is open source, free software, and contains the same subroutines we use for impact monitoring†. You can download it, compile on your own computer and run it on the same data we have, and reproduce the same results; there are people who have actually done this, as well as comparing with their own software $\neq$. Thus there is secret neiter on the data, nor on the methods, nor on the results.

Once we have something which is out, there is typically a short time before the press notices it. Our sites, NEODyS ans Sentry, are monitored every 5 minutes to look for new and/or changed announcements on possible impact; e.g., such continous control is performed by the MPC, for obvious reasons, and by Bill Allen $\uparrow$ who keeps a record so accurate of all the changes in our sites that we actually use his web site when we need a record of our previous postings. The response by the media may be slow or even null in some cases, e.g., at the worst moment of the (99942) Apophis crisis (26 December 2004) there was the tsunami and the $1 / 37$ impact probability was essentially not reported by the media. In other cases, however, depending upon the hour in some time zone and upon the internal logic of the media, there can be a significant response in few hours.

This is the situation which has to be the main concern of the Impact threat committee: the information on a possible impact is not secret, it is out on the web and nobody has noticed it yet. During that short time span we need to know what to say to better convey the information to the general public, and in extreme cases to the authorities if they need to act for mitigation, as it has not happened yet. My understanding is that this is the main function of the new advisory group.

EngVolD: They have to keep the SG informed, but they are the best placed persons to decide how to pass the information to the press.

$\dagger$ http:newton.dm.unipi.it/orbfit/

$\ddagger$ For example, I. Wlodarczyk in Poland.

I http://www.hohmanntransfer.com 
MARSDEN: If we discover something, probably very small, which is going to hit in a matter of days, that will become obvious at the MPC. We hope it does not happen, but if it happens, it has to be handled in an appropriate way.

HARRIS: Current surveys have the capability to find objects with diameter few tens of meters, the next generation surveys could find meteoroids of few meters, with an impact rate on Earth's atmosphere of the order of one per year. Thus there is a significant probability that they would discover one of these while it is arriving.

MORRISON: The cases that will most likely cause difficulty in communicating with the public are those of impacts decades away in time, that may raise to a probability level of a few percent and then go away, rather than the rare cases in which a very small impactor is discovered hours or days before it impacts.

VALSECCHI: Questions related to the announcement dilemma are possibly not the hardest problem our community faces; rather, we have not yet been able to convince responsible people outside the astronomical community that what we have put in place (i.e., NEO discovery, impact monitoring and space missions to test mitigation techniques) is just the first link in the chain of actions to prevent consequences of impacts, and that all the other links, involving civil protection, policy issues, social issues, legal issues etc., have still to be put in place.

Andersen: The IAU General Secretary, even if not a NEO-specialist, will make sure that the IAU has appropriate structures in place to deal with the NEO issue; it is clear that many, if not most, countries, have not the foggiest idea of what to do in case the announcement of a real impact were made, and that is where you need the IAU GS, who can speak in the name of the world astronomers, providing mankind the relevant information. It is then to mankind to find out what to do. 